\title{
Efficacy of Gamma Knife Radiosurgery in Refractory Obsessive-Compulsive Disorder: An Indian Experience
}

\author{
Sanjeev Pattankar ${ }^{1}$ Milind Sankhe ${ }^{1}$ Kersi Chavda ${ }^{2}$ \\ ${ }^{1}$ Department of Neurosurgery and Gamma Knife Radiosurgery, \\ Parmanand Deepchand Hinduja Hospital \& Medical Research Center, \\ Mumbai, Maharashtra, India \\ 2 Department of Psychiatry, Parmanand Deepchand Hinduja Hospital \\ \& Medical Research Center, Mumbai, Maharashtra, India \\ Address for correspondence Milind Sankhe, MBBS, MS, MCh, FRCS \\ (SN), Department of Neurosurgery and Gamma Knife Radiosurgery, \\ Parmanand Deepchand Hinduja Hospital \& Medical Research Center, \\ Veer Savarkar Marg, Mahim, Mumbai, Maharashtra 400016, India \\ (e-mail: milsankhe@gmail.com).
}

J Neurosci Rural Pract 2022;13:23-31.

\section{Abstract \\ Keywords \\ - Gamma Knife radiosurgery \\ - refractory OCD \\ - obsessive-compulsive disorder \\ - long-term outcome}

Background Obsessive-compulsive disorder (OCD) is a chronic debilitating psychiatric condition with adverse impact on patient's sociooccupational health. Refractoriness to pharmacotherapy and psychotherapy is not uncommon. Gamma Knife radiosurgery (GKRS) is the comprehensively used and reviewed treatment modality in refractory OCD worldwide. In India, the past two decades of increasing GKRS availability has failed to create the necessary local awareness of its usefulness in refractory OCD. Limited native literature deepens the problem.

Objective To analyze our experience with GKRS in refractory OCD, and report the safety and efficacy/long-term outcome in patients using the Yale-Brown ObsessiveCompulsive Scale (Y-BOCS).

Materials and Methods A retrospective review of patients receiving GKRS for refractory OCD between 2000 and 2020 was carried out. Case files of the eligible $(n=9)$ patients were reviewed for clinical, radiotherapeutic, and outcome data. Additionally, patients were contacted via telephone to enquire about their experiences, and to obtain retroactive consent for GKRS in June 2021. Information obtained was collated, computed, and analyzed.

Results Male-to-female sex ratio was 8:1. Mean age at the time of GKRS and mean duration of OCD prior to GKRS was $30.1 \pm 9.4$ and $10.2 \pm 5.8$ years, respectively. Mean baseline Y-BOCS score was $29.6 \pm 4.7$. Our first patient received cingulotomy, while the rest underwent anterior capsulotomy. Median margin dose (50\% isodose) was $70 \mathrm{~Gy}$. Also, $23.8 \pm 7.7$ was the mean Y-BOCS score at the last follow-up (median $=30$ months). Overall, $44.4 \%$ patients showed full/partial response ( $\geq 25 \%$ reduction in $Y$ BOCS score) at the last follow-up. In anterior capsulotomy (eight patients), patients with moderate/severe OCD showed better response ( $4 / 5$ responders) than those with extreme OCD (0/3 responders). Single case of cingulotomy resulted in no response published online January 5, 2022
DOI https://doi.org/

10.1055/s-0041-1740453. ISSN 0976-3147. (c) 2022. Association for Helping Neurosurgical Sick People. All rights reserved.

This is an open access article published by Thieme under the terms of the Creative Commons Attribution-NonDerivative-NonCommercial-License, permitting copying and reproduction so long as the original work is given appropriate credit. Contents may not be used for commercial purposes, or adapted, remixed, transformed or built upon. (https://creativecommons.org/ licenses/by-nc-nd/4.0/)

Thieme Medical and Scientific Publishers Pvt. Ltd., A-12, 2nd Floor, Sector 2, Noida-201301 UP, India 
( $<25 \%$ reduction in Y-BOCS score). No adverse radiation effects were noted. Also, $55.6 \%$ patients gave retroactive consent telephonically.

Conclusion GKRS is a safe and effective noninvasive treatment modality for refractory OCD. Ventral anterior capsule is the preferred target. Maximum radiation doses of 120 to 160 Gy are well tolerated. Extremely severe OCD cases fared poorer. Proper awareness about the availability and efficacy of GKRS in refractory OCD is required in India.

\section{Key Message}

GKRS is a safe and effective noninvasive treatment modality for refractory OCD. Proper awareness about the availability and efficacy of GKRS in refractory OCD is required in India.

\section{Introduction}

Obsessive-compulsive disorder (OCD) is a chronic debilitating psychiatric condition characterized by obsessions (recurring intrusive thoughts, ideas, or images) and/or compulsions (recurring ritualized behaviors) which are anxiety inducing, time-consuming, and result in poor sociooccupational health. ${ }^{1}$ With 2 to $3 \%$ of prevalence seen in general population, OCD is the fourth most commonly diagnosed psychiatric condition worldwide. ${ }^{2}$ Advancements in pharmacotherapeutics and psychotherapy (first-line treatments) have failed to reduce the refractoriness (10-20\%) in OCD patients. ${ }^{3}$ Chronicity, young age at diagnosis, and treatment refractoriness negatively impact patient's functional status which, in severe cases, may result in suicidal ideations and premature death. ${ }^{4}$ Hence, it is imperative to treat OCD cases timely and appropriately to keep in check its socioeconomic burden on the society.

Since the middle of 20th century, furtherance in the principles of stereotaxy and neuroimaging, along with the betterment in the understanding of the hyperactive orbitofronto-striato-thalamo-cortical circuitry, has led to neurosurgical procedures being increasingly resorted to in the management of refractory OCD patients. ${ }^{5,6}$ At present, a range of ablative techniques are available to target regions like anterior limb of internal capsule (ALIC; anterior capsulotomy, preferred with the largest evidence), anterior cingulate gyrus/cingulum bundle (cingulotomy), ventral corticostriatal tracts (subcaudate tractotomy), and others. ${ }^{7}$ Though the initially developed invasive stereotactic radiofrequency thermocoagulation techniques are still in practice for selected cases, their noninvasive counterparts, like Gamma Knife radiosurgery (GKRS) and magnetic resonance-guided focused ultrasonography (MRgFUS), have taken over as the preferred treatment modalities, especially for anterior capsulotomies. ${ }^{8,9}$ Additionally, theoretically superior neuromodulation techniques, like deep brain stimulation (DBS), are yet to prove their worthiness in refractory $O C D$, justifying their high cost and associated multiple invasive procedures (electrode replacement/battery changes) by achieving better targeting and/or outcome. ${ }^{10}$

GKRS lesioning has been increasingly employed in treating refractory OCD in the past three decades. ${ }^{8}$ Evidencebased tailoring of the GKRS radiotherapeutic profile and targeting, has led to the expanding of its therapeutic window, allowing even moderate yet disabling refractory OCD cases to safely reap the benefits of GKRS lesioning. As GKRS acts by both direct modulation of pathological neural pathways and in synergy to the ongoing pharmacological or psychological therapies, the patients should clearly be explained about the expected outcomes (especially, need for continuing first-line therapies, $50-75 \%$ response rates, and low but existing risk of adverse effects). In India, the past two decades of increasing GKRS availability has failed to create the necessary local awareness of its usefulness in refractory OCD. No native literature is available on the experiences with GKRS in refractory OCD cases, except a single case report about late radiation necrosis post-GKRS capsulotomy. ${ }^{11}$ In this study, we aim to analyze our center's experience with GKRS in refractory OCD and report the safety, and efficacy/long-term outcome in patients using the Yale-Brown Obsessive Compulsive Scale (Y-BOCS).

\section{Materials and Methods}

\section{Study Design}

This is a retrospective study including all the consecutive cases $(n=9)$ of refractory OCD treated by GKRS lesioning, between 2000 and 2020, at the Parmanand Deepchand Hinduja hospital in Mumbai. Case files of the eligible patients were reviewed for clinical, radiotherapeutical, and outcome data. Additionally, patients were contacted via telephone to enquire about their experiences, and to obtain retroactive consent for GKRS in June 2021. Retroactive consent was defined as the hypothetical continued willingness of the patient to uphold the pre-GKRS lesioning consent given by self, having experienced its effects. Information obtained was collated, computed, and analyzed. Means, medians, and standard deviations were calculated for various continuous variables. Standard statistical tests were used applied and a $p$-value of $<0.05$ was considered significant.

\section{Patient Selection}

Every patient's suitability for GKRS was jointly decided by a multidisciplinary team comprising of psychiatrists, clinical 
psychologists, and neurosurgeons. Only those patients with chronic ( $>5$ years), excessively time consuming, sociooccupationally impairing, and refractory OCD with Y-BOCS score $\geq 24$ (severe OCD) and without any structural brain abnormalities were considered for GKRS lesioning. ${ }^{8}$ One exception to this rule of minimum Y-BOCS score was made for patient no. 3 treated with GKRS, as he was fulfilling every other criterion and was insistent on receiving GKRS to be able to continue his higher education. Refractoriness of the OCD was determined by an independent psychiatrist, as failure of at least three adequate trials of serotonin reuptake inhibitors (including clomipramine), an adequate trial of psychotherapy, and/or ablative neurosurgical procedure (other than GKRS). Detailed consultations regarding expectations from GKRS lesioning, and possible outcomes were done with the patient and family before obtaining informed consent in every case.

\section{Clinical Assessment and Follow-up}

Every patient receiving GKRS for refractory OCD was subjected to a fresh neurological and neuropsychological examinations prior to the procedure. Severity of the refractory OCD was quantified based on Y-BOCS test, administered by an experienced clinical psychologist. Interpretation of the obtained Y-BOCS scores was as follows: 0 to 7 indicating subclinical symptoms, 8 to 15 mild symptoms, 16 to 23 moderate symptoms, 24 to 31 severe symptoms, and 32 to 40 extreme symptoms. ${ }^{12}$ Response to GKRS lesioning at last follow-up was classified based on change in Y-BOCS scores as follows: "full responder" if $\geq 35 \%$ reduction seen in Y-BOCS score, "partial responder" if 25 to $34 \%$ reduction seen in Y-BOCS score, and "nonresponder" if $<25 \%$ reduction seen in Y-BOCS score. ${ }^{13-16}$ Patients with both full and partial responses were considered responders. All the patients were followed-up at every 6 months in the first-year post-GKRS, and yearly thereafter. Each follow-up involved a fresh clinical psychology and a psychiatric consultation, with magnetic resonance imaging (MRI) brain in the first two follow-ups to confirm the GKRS lesioning status.

\section{Gamma Knife Radiosurgery Procedure}

On the day of the procedure, the Leksell frame (model G, Elekta) was fixed to the head of the patient under local anesthesia. Using thin ( $1 \mathrm{~mm}$ ) volumetric MRI T1-weighted images and T2-weighted axial and coronal slices, target sites were marked by the neurosurgeon with the help of a radiologist and a radiation physicist. Since ours' was the first GKRS center in the entire South Asian region and refractory OCD referrals were limited, our targeting techniques kept evolving with our own experiences, and with the emerging global consensus. Our first patient underwent a cingulotomy, wherein we delineated the anterior portion of the cingula from the level of the anterior commissure and placed two shots/isocenters to target each delineated area using 4-mm collimators. Rest of the patients $(n=8)$ underwent an anterior capsulotomy, wherein variable number of shots/isocenters ( 2 to 5 ) were placed using 4-mm collima- tors, targeting the midputaminal point of the ALIC on each side, at the most ventral portion. Majority of our anterior capsulotomies involved either triple shot/isocenter lesioning (five patients) or double-shot/isocenter lesioning (two patients), both targeting the ventral ALIC. Only one patient received more than three shots/isocenters targeting (nine shots bilaterally) the entire ALIC, based on an individualized decision between the neurosurgeon, psychiatrist, and the patient. Presently, we follow the double-shot/isocenter targeting technique to midputaminal ventral ALIC, similar to gamma ventral capsulotomy (GVC) used worldwide. Maximum radiation dose ( $100 \%$ isodose) varied between 120 to $160 \mathrm{~Gy}$. The Leksell frame was removed postprocedure and sterile dressings were applied to the pin sites. The patients were discharged home on first postoperative day. - Fig. 1 shows two serial follow-up MRI images post-GKRS anterior capsulotomy, including GKRS planning, in patient no. 3 .

\section{Results}

\section{Demographics}

Male-to-female sex ratio was 8:1. Mean age at the time of GKRS and mean duration of OCD prior to GKRS was $30.1 \pm 9.4$ and $10.2 \pm 5.8$ years, respectively. Comorbid psychiatric conditions like generalized anxiety disorder (55.6\%), major depression (44.4\%), and suicidal ideations (33.3\%) were seen. Most common types of obsessions and compulsions noted in the study group were contamination and cleaning or washing, respectively. Mean baseline Y-BOCS score was $29.6 \pm 4.7$. All the patients had received multiple unsuccessful trials of both pharmacotherapy and psychotherapy preGKRS, with one patient having history of failed thermocingulotomy. Other details like educational, employment, and marital status of the study group have been described in -Table 1.

\section{Radiotherapeutic Profile and Outcome Data}

Our first patient received cingulotomy, while the rest underwent anterior capsulotomy. Median margin dose (50\% isodose) was $70 \mathrm{~Gy}$ (range $=60-80 \mathrm{~Gy}$ ). Total shots/isocenters used to deliver the radiation dose ranged from 4 to 9 (median =6). $23.8 \pm 7.7$ was the mean Y-BOCS score at the last follow-up (median $=30$ months, range $=3-192$ months). Overall, $44.4 \%$ patients showed full/partial response ( $\geq 25 \%$ reduction in Y-BOCS score) at the last follow-up. Single case of cingulotomy resulted in no response $(<25 \%$ reduction in Y-BOCS score; - Table 2 ).

On subgroup analysis in anterior capsulotomy group (eight patients), 50\% full/partial response was seen. Patients with moderate/severe OCD showed better response $(4 / 5$ responders) than those with extreme OCD $(0 / 3$ responders; $p=0.02$ ). Duration of OCD prior to GKRS ( $<10$ or $\geq 10$ years) and maximum radiation dosage received showed no meaningful association with response rates. Responders in tripleand double-shot/isocenter lesioning were two out of five patients, and one out of two patients, respectively. Single multishot lesioning ( $>3$ shots/isocenters) resulted in partial response at last follow-up. 


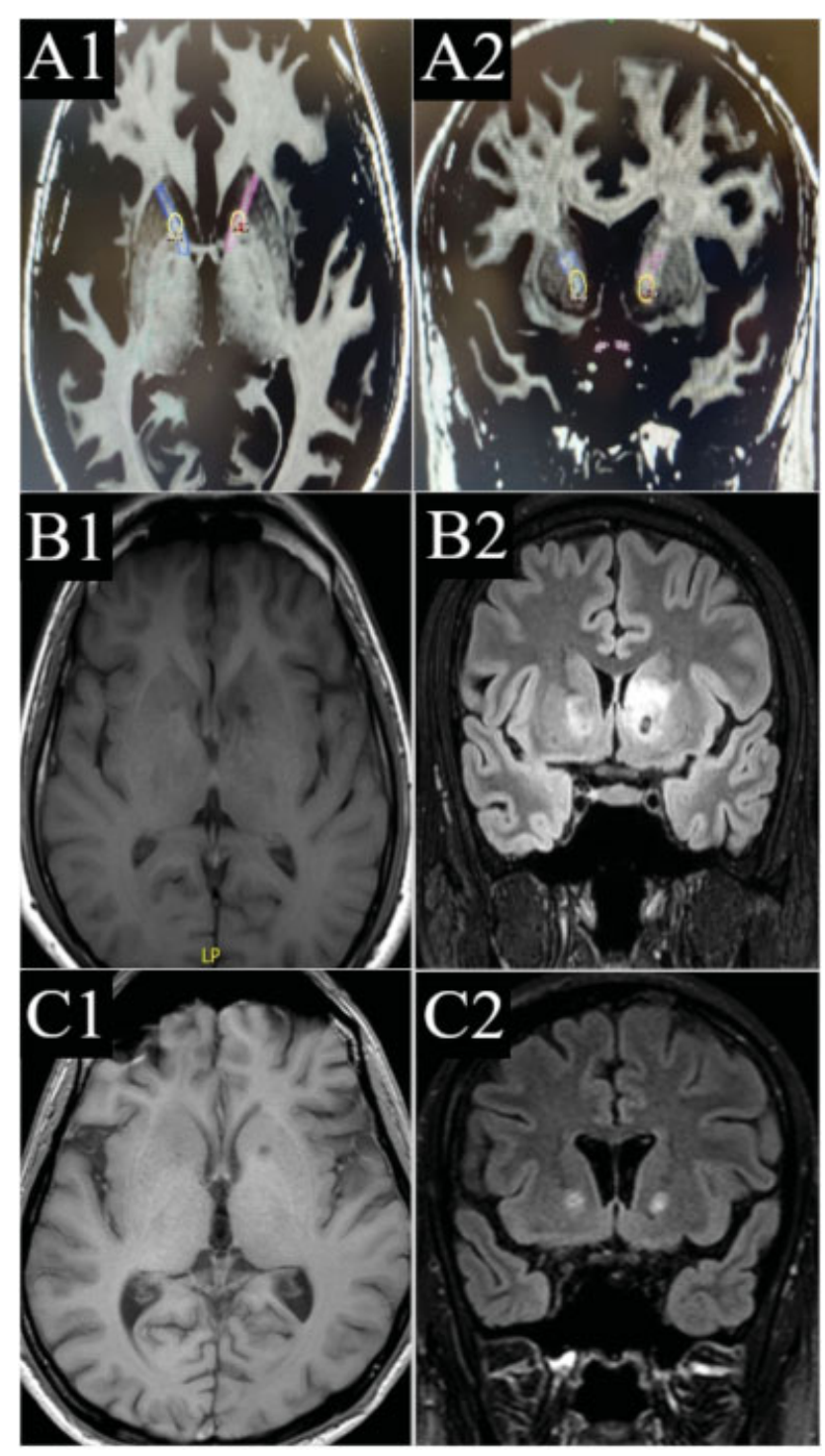

Fig. 1 (A1-C2) Serial MRI images belonging to patient no. 3, who underwent GKRS anterior capsulotomy. (A1-A2) Axial (A1) and coronal (A2) T1-weighted images showing marked anterior limb of internal capsule (ALIC) bilaterally (blue line-right ALIC; purple line-left ALIC), with ventral mid-putaminal ALIC receiving 70 Gy margin dose (50\% isodose line - yellow) via 3 shots/isocenters. (B1-B2) Axial (B1) T1-weighted and coronal (B2) FLAIR images showing post-GKRS lesioning status at 6 months. Bilateral hypointense ALIC lesions (left more than right) can be appreciated. Additionally, FLAIR images show surrounding hyperintense post-GKRS effects. (C1-C2) Axial (B1) T1weighted and coronal (B2) FLAIR images showing post-GKRS lesioning status at 12 months. Resolution of hyperintensity surrounding ALIC lesions seen on FLAIR images. FLAIR, fluid-attenuated inversion recovery; GKRS, Gamma Knife radiosurgery; MRI, magnetic resonance imaging.

MRI imaging to confirm post-GKRS lesioning status was available in eight out of nine patients, with appropriately placed lesions as per GKRS planning in seven out of eight patients. Post-GKRS treatment modalities employed in the study group included pharmacotherapy (55.6\%), psychotherapy $(22.2 \%)$, thermocapsulotomy (11.1\%), and transcranial direct current stimulation (11.1\%). Employment status remained the same till the last follow-up, except for the two students in the study group who finished their gradu-
Table 1 Demographics and clinical data of the study group

\begin{tabular}{|l|l|}
\hline Characteristic & $\begin{array}{l}\text { Frequency/ } \\
\text { Mean } \pm \text { SD/n (\%) }\end{array}$ \\
\hline Patients (n) & 9 \\
\hline Age at GKRS (y) & $30.1 \pm 9.4$ \\
\hline Sex ratio (male:female) & $8: 1$ \\
\hline Duration of OCD prior to GKRS (y) & $10.2 \pm 5.8$ \\
\hline Comorbid conditions & \\
\hline Generalized anxiety disorder & $5(55.6)$ \\
\hline Major depression & $4(44.4)$ \\
\hline Suicidal thoughts & $3(33.3)$ \\
\hline Types of obsessions & \\
\hline Contamination & $7(77.8)$ \\
\hline Doubting & $5(55.6)$ \\
\hline Ordering & $2(22.2)$ \\
\hline Aggressive & $4(44.4)$ \\
\hline Sexual & $3(33.3)$ \\
\hline Types of compulsions & \\
\hline Cleaning/washing & $7(77.8)$ \\
\hline Checking & $4(44.4)$ \\
\hline Ordering/arranging & $4(44.4)$ \\
\hline Scrupulosity & $3(33.3)$ \\
\hline Hoarding & $2(22.2)$ \\
\hline Education status & \\
\hline High school & $3(33.3)$ \\
\hline Undergraduate & $5(55.6)$ \\
\hline Postgraduate & $1(11.1)$ \\
\hline Employment status prior to GKRS & \\
\hline Employed & $3(33.3)$ \\
\hline Unemployed & $3(33.3)$ \\
\hline Student & $2(22.2)$ \\
\hline Housewife & $1(11.1)$ \\
\hline Marital status prior to GKRS & \\
\hline Unmarried & $7(77.8)$ \\
\hline Married & $2(22.2)$ \\
\hline Pre-GKRS treatment modalities & $9(100)$ \\
\hline Pharmacotherapy & \\
\hline Psychotherapy & (100) \\
\hline Thermocingulotomy & \\
\hline Baseline Y-BOCS score prior to GKRS & \\
\hline Srens & \\
\hline
\end{tabular}

Abbreviations: GKRS, Gamma Knife radiosurgery; OCD, obsessive compulsive disorder; SD, standard deviation; Y-BOCS, Yale-Brown Obsessive Compulsive Scale.

ations and went on to be successfully employed, albeit with some limitations. No major adverse radiation effects were noted till the last follow-ups. Normal neurological status was seen in all the patients. Two patients reported an increase in 
Table 2 Radiotherapeutic profile and outcome data in the study group

\begin{tabular}{|c|c|}
\hline Characteristic & $\begin{array}{l}\text { Frequency/n } \\
\text { (\%)/median } \\
\text { (range) }\end{array}$ \\
\hline Sessions of GKRS (n) & 9 \\
\hline \multicolumn{2}{|l|}{ Targets of GKRS } \\
\hline Cingulotomy & $1(11.1)$ \\
\hline Anterior capsulotomy & $8(88.9)$ \\
\hline Margin dose ( $50 \%$ isodose) in Grays & $70(60-80)$ \\
\hline Shots/isocenters & $6(4-9)$ \\
\hline Follow-up after GKRS (mo) & $30(3-192)$ \\
\hline Y-BOCS score at last follow-up (mean \pm SD) & $23.8 \pm 7.7$ \\
\hline \multicolumn{2}{|l|}{$\begin{array}{l}\text { Post-GKRS outcome in anterior } \\
\text { capsulotomy (patients }=8 \text { ) }\end{array}$} \\
\hline $\begin{array}{l}\text { Full/partial responders ( } \geq 25 \% \text { reduction } \\
\text { in Y-BOCS score) }\end{array}$ & $4(50)$ \\
\hline $\begin{array}{l}\text { Nonresponders ( }<25 \% \text { reduction in } \\
\text { Y-BOCS score) }\end{array}$ & $4(50)$ \\
\hline \multicolumn{2}{|l|}{$\begin{array}{l}\text { Post-GKRS outcome in cingulotomy } \\
\text { (patients }=1 \text { ) }\end{array}$} \\
\hline $\begin{array}{l}\text { Nonresponder ( }<25 \% \text { reduction in } \\
\text { Y-BOCS score) }\end{array}$ & $1(100)$ \\
\hline Adverse radiation effects & $0(0)$ \\
\hline \multicolumn{2}{|l|}{ Post-GKRS treatment modalities } \\
\hline Pharmacotherapy & $5(55.6)$ \\
\hline Psychotherapy & $2(22.2)$ \\
\hline Thermocapsulotomy & $1(11.1)$ \\
\hline Transcranial direct current stimulation & $1(11.1)$ \\
\hline \multicolumn{2}{|l|}{ Employment status at last follow-up } \\
\hline Employed & $5(55.6)$ \\
\hline Unemployed & $3(33.3)$ \\
\hline Housewife & $1(11.1)$ \\
\hline \multicolumn{2}{|l|}{ Marital status at last follow-up } \\
\hline Unmarried & $7(77.8)$ \\
\hline Married & $2(22.2)$ \\
\hline $\begin{array}{l}\text { Retrospective consent for GKRS obtained } \\
\text { telephonically }\end{array}$ & $5(55.6)$ \\
\hline
\end{tabular}

Abbreviations: GKRS, Gamma Knife radiosurgery; SD, standard deviation; Y-BOCS, Yale-Brown Obsessive Compulsive Scale.

weight of $>5 \mathrm{~kg}$ in the anterior capsulotomy group. None had reported any personality changes.

\section{Individual Case Details}

Detailed demographic and radiotherapeutic characteristics, along with outcome and follow-up data of the study group has been presented in -Table 3. Some of the unique patient profiles and their details are as follows:

- Patient no. 3 was a case of posttraumatic (diffuse axonal injury) refractory OCD with moderate severity (Y-BOCS
=19). Patient was a medical doctor himself and was severely debilitated by the disease itself, as well as the side effects of prescribed pharmacotherapeutics. In view of being able to continue his higher studies, patient was insistent on GKRS anterior capsulotomy, very well understanding its risk-to-benefit ratio. Patient withstood the procedure well (-Fig. 1) and is completely off the medications with manageable OCD symptoms.

- Patient no. 4 was the only patient in the study group with prior nonresponse to an ablative procedure (thermocingulotomy). He remained nonresponder to GKRS anterior capsulotomy till last follow-up. So, careful counseling prior to GKRS lesioning is required in patients who are nonresponders to a radiologically successful ablative procedure in the past.

- Patient no. 5 was a nonresponder at 1-year follow-up post-GKRS anterior capsulotomy, with MRI imaging showing radiologically failed anterior capsulotomy status as shown in -Fig. 2A. Patient and the family were counselled regarding the same and were given an option of undergoing a thermocapsulotomy via radiofrequency ablation. Patient consented for the same, and the postlesioning status was as seen in the MRI in - Fig. 2B. This resulted in tremendous improvement in his Y-BOCS $(=18)$ scores and is continuing to be off medications with manageable OCD symptoms.

\section{Retroactive Consenting}

In June 2021, all the patients were contacted telephonically to enquire about their expectations and practical experiences with GKRS lesioning in treating their OCDs. Five (55.6\%) patients said that their GKRS results were significant enough to warrant a retroactive consent for the same. Patient no. 5, who received a thermocapsulotomy post-GKRS nonresponse, gave a retroactive consent for the former type of capsulotomy. And four (44.4\%) patients said that they would recommend GKRS lesioning to other fellow OCD patients.

\section{Discussion}

In this retrospective study, we present the experience of the first GKRS center established in the South Asian region, in treating refractory OCD. Our targeting techniques kept evolving in the last two decades, with our own experiences, and with the emerging global consensus. Hence, a spectrum of radiotherapeutic profiles and targeting techniques can be seen in our study group (-Table 3 ). Despite these dissimilarities in the study group, the observations made in our results section reinforce the robust safety (no major adverse radiation effects) and fair efficacy/long-term outcome (44.4\% overall; $50 \%$ in anterior capsulotomies) of GKRS lesioning in refractory OCD cases. Extremely severe (Y-BOCS $\geq 32$ ) OCD cases fared poorer compared to moderately/severely (YBOCS: 16-31) affected OCD cases which is a unique finding. A good proportion of our patients extending retroactive consent (55.6\%) for GKRS lesioning is an excellent rebuttal to the naysayers in the field. More native literature from various GKRS centers in India, regarding their experiences 


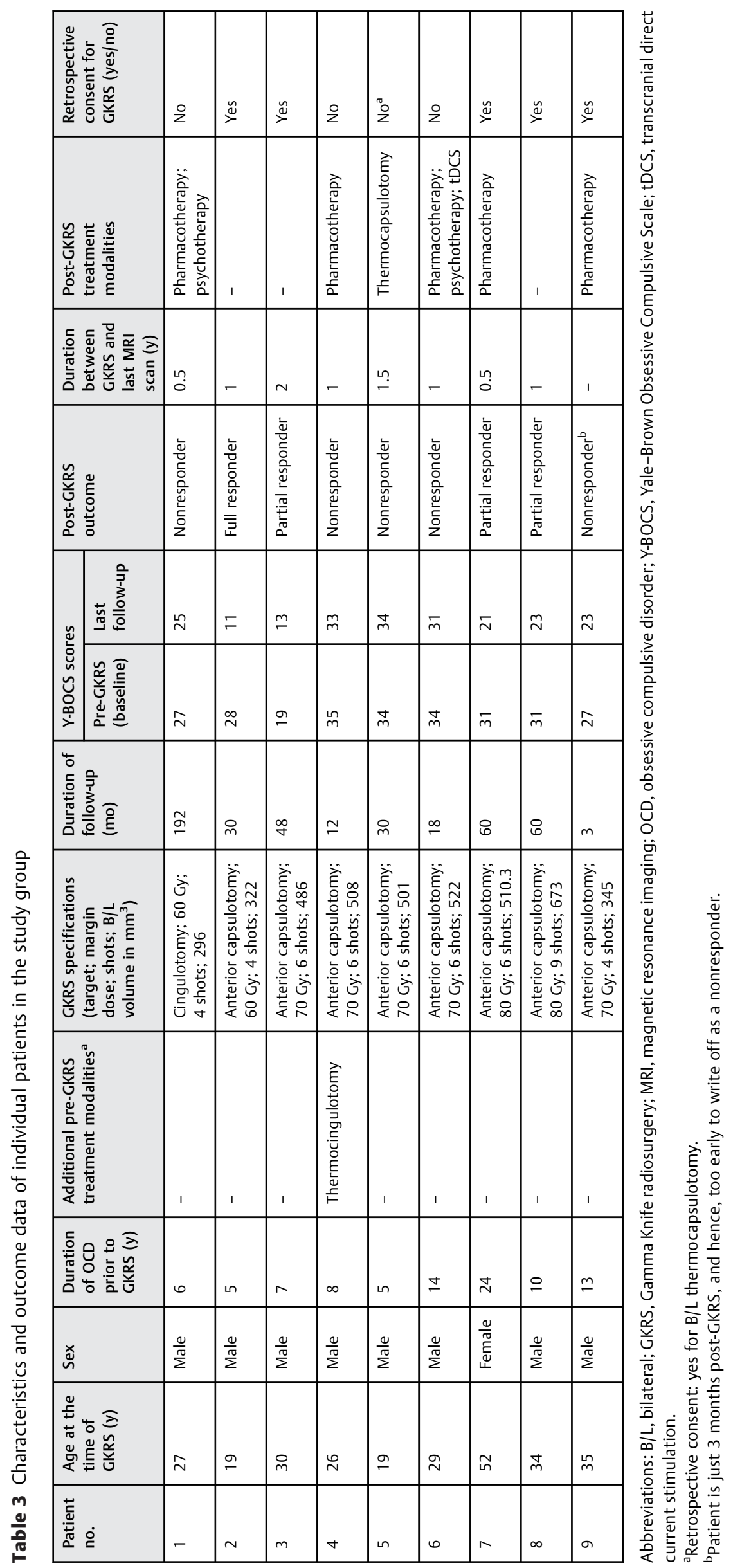




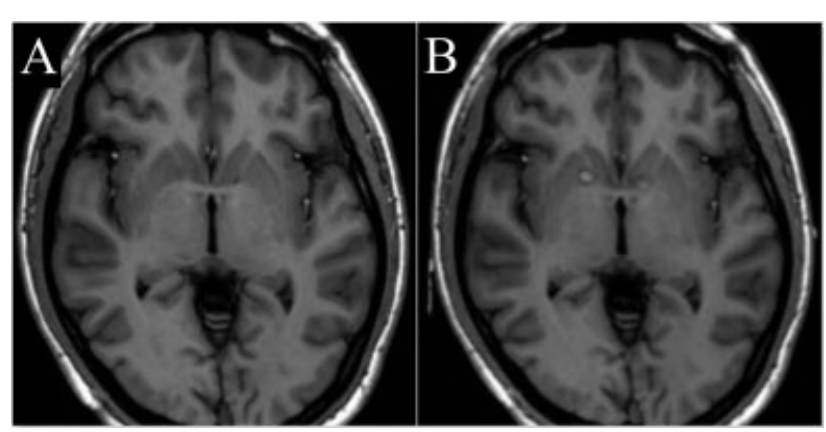

Fig. 2 (A, B) Serial MRI axial T1-weighted images in patient no. 5. (A) 1-year follow-up MRI post-GKRS anterior capsulotomy, showing radiologically failed anterior capsulotomy status. Subsequently, patient received a bilateral thermocapsulotomy via radio-frequency ablation. (B) MRI images showing post thermo-capsulotomy status. Bilateral ALIC lesions can be appreciated. ALIC, anterior limb of internal capsule; GKRS, Gamma Knife radiosurgery; MRI, magnetic resonance imaging.

with refractory $O C D$, will bring about the required awareness.

\section{Neurosurgery in Refractory Obsessive-Compulsive Disorder}

Neurosurgery has a last-tier role to play in the management of refractory OCD. It is prudent to mention the contributions of Sao Paulo group in championing the strict inclusion/exclusion criteria for neurosurgery in refractory OCD. ${ }^{8,16}$ They advocate neurosurgical treatment only if patients have the following: (1) a main diagnosis of OCD (troublesome OCD symptoms, if comorbid axis-I or -II disorders present); (2) Y-BOCS score of $\geq 28$ or $\geq 14$ if only obsessions or only compulsions are present, with OCD being extremely time consuming or impairing in all the cases; (3) $\geq 5$ years of severe OCD symptoms despite adequate treatment trials; (4) refractoriness defined as insufficient response to $\geq 3$ adequate trials with serotonin reuptake inhibitors (at least one with clomipramine), $\geq 2$ adequate augmentation strategies (typical/atypical antipsychotic drugs or clomipramine), and $\geq 20$ hours of OCD-specific behavioral therapy. ${ }^{8}$

Procedures available are of following two types: ablative and neuromodulatory. ${ }^{5}$ More commonly used are the ablative procedures which are permanent lesioning techniques, employing either invasive (electrocautery/radiofrequency) or noninvasive (GKRS and MRgFUS) lesion generators. ${ }^{5,8,10}$ No single clinical study is available till date, comparing the safety and efficacy/long-term outcome of various ablative techniques, that is, anterior capsulotomy, cingulotomy, subcaudate tractotomy, and limbic leucotomy (a combination of anterior cingulotomy and subcaudate tractotomy). A recent meta-analysis published by Lai et al, comparing various ablative procedures and their outcomes; a pooled group of 459 patients found response rates of 59,47 , and $36 \%$, respectively, with anterior capsulotomy, limbic leucotomy, and cingulotomy. ${ }^{7}$ This reinforces the selective preference enjoyed by the anterior capsulotomy in treating refractory OCD worldwide. Though GKRS remains the most widely used and thoroughly studied noninvasive ablative technique for now, MRgFUS is rapidly gaining ground on the basis of its ability to monitor in real-time the lesion formation and avoiding exposure to ionizing radiations. ${ }^{10}$

DBS is the Food and Drug Administration (FDA) approved (on humanitarian grounds) neuromodulatory procedure available for refractory OCD. ${ }^{5,10}$ Surgeons are increasingly lured by its adjustability and reversibility compared to permanent ablative procedures, even though DBS has some major inherent disadvantages like life-long follow-up, multiple surgeries, and higher cost. ${ }^{10}$ Commonly used DBS targets include ALIC, ventral capsule/ventral striatum, nucleus accumbens, and others. The only meta-analysis (Kumar et al) available on the comparative effectiveness of ablative procedures and DBS in refractory OCD has been a myth buster. Across 681 cases in 56 studies, ablative procedures had a better Y-BOCS scores reducing ability of $50.4 \%$ (vs. $40.9 \%$ of DBS). Concluding that in its current form, the theoretical superiority of DBS over an ablative procedure does not appear to positively affect its outcome profile. ${ }^{10}$

\section{Gamma Knife Radiosurgery Lesioning in Refractory Obsessive-Compulsive Disorder}

Since its introduction as a noninvasive lesioning technique for ablative OCD procedures by Karolinska Institute in 1976, GKRS lesioning has seen a significant evolution in its targeting techniques and radiotherapeutic profiling. GKRS in refractory OCD is synonymous with GKRS anterior capsulotomy, as the interest in targeting other sites (like cingulotomy) with GKRS never picked up. ${ }^{8}$ Though anterior capsulotomies were started with higher 180 to $200 \mathrm{~Gy}$ radiation doses, the associated adverse radiation effects, and absence of better outcome advantage, compelled the reduction of radiation doses to 120 to $160 \mathrm{~Gy} .{ }^{8}$ With regard to evolution of shots/isocenters placement in anterior capsulotomies, the journey of GKRS started with the middle part of the ALIC (single shot), going on to include the entire ALIC (three shots), and, more recently, progressing to target only the ventral portion of the capsule (single or double shots) called gamma ventral capsulotomy (GVC). ${ }^{17}$ The meta-analysis by Lai et al also analyzed effectiveness of various types of lesioning in ALIC and found response rates of 58,67 , and $80 \%$ in the groups with lesions involving the entire ALIC (three shots), the ventral third of ALIC (double shots), and the ventral capsule (single shot), respectively. ${ }^{7}$ This justifies the increasing trend of GVC seen worldwide in refractory OCD management. Furthermore, GVC is the only ablative procedure whose safety and efficacy has been validated by a double-blind, sham-controlled, randomized clinical trial. ${ }^{16}$ Additionally, there is an increasing evidence to suggest the usefulness of diffusion tensor imaging (DTI) in delineating the orbitofrontal cortex (OFC) bundles in ALIC, and the potential help it provides in shots/isocenters placement for better outcome in GVC. ${ }^{18}$ Graph in - Fig. 3 delineates the YBOCS-based outcome seen in various studies published on GKRS anterior capsulotomy. ${ }^{14-17,19-24}$ Though the study groups have varying spectrum of demographical, clinical, and radiotherapeutic profiles, the long-term response rates 


\section{Various studies on outcome of GKRS in OCD: Y-BOCS based}

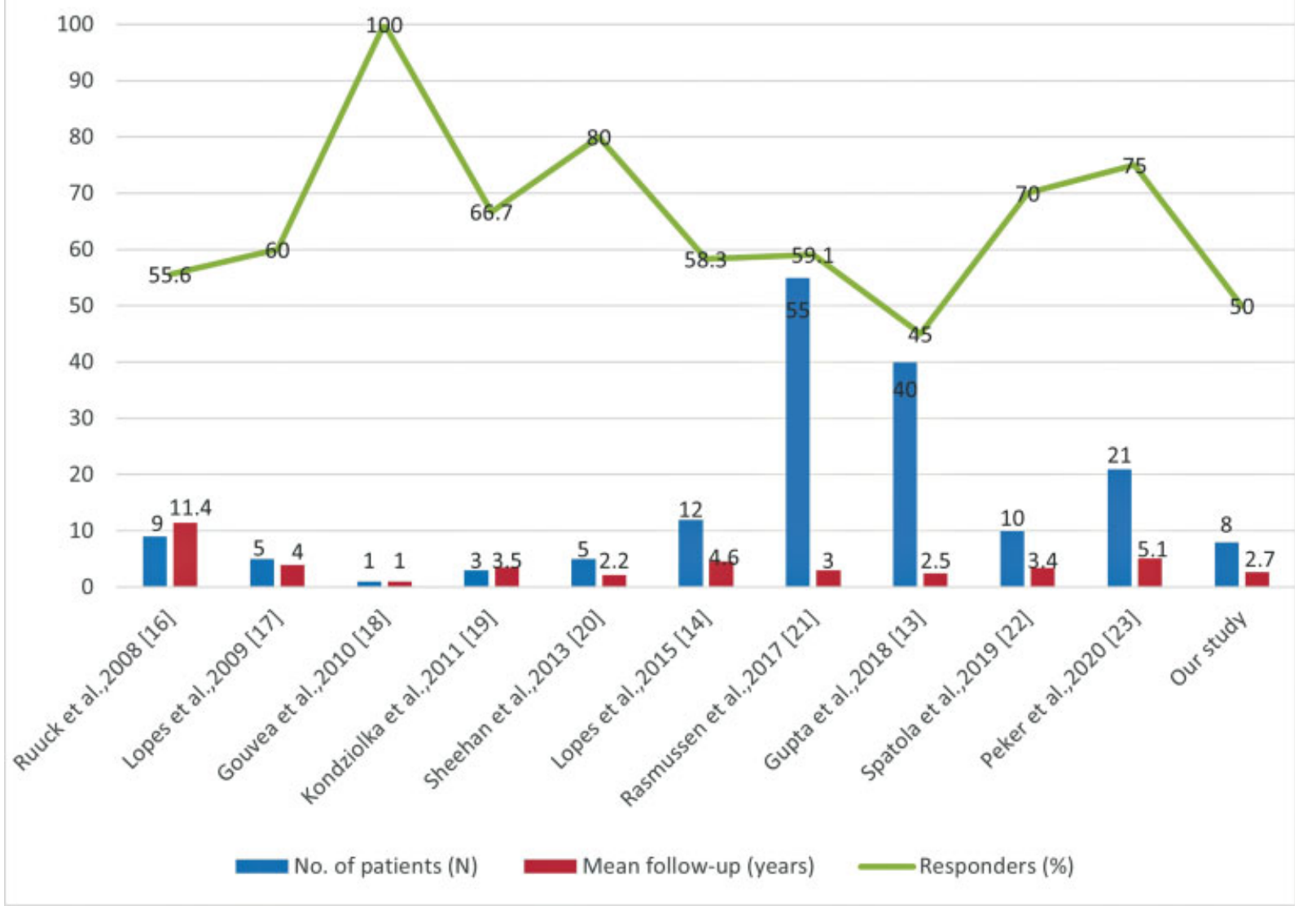

Fig. 3 Graphical representation of various studies (Y-BOCS based) available on the outcome of GKRS anterior capsulotomy in refractory OCD. Note: only our center's results of GKRS anterior capsulotomy are depicted here. GKRS, Gamma Knife radiosurgery; OCD, obsessive-compulsive disorder; Y-BOCS, Yale-Brown Obsessive-Compulsive Scale.

are uniformly in the range of one-half to two-thirds in all the studies, including the present one. ${ }^{8}$

About $90 \%$ of adverse radiation effects are transient and manageable which includes headaches, cognitive deficits, and behavior problems. Minority of patients end up with permanent deficits like personality changes, as well as brain cysts or brain edema. ${ }^{7}$ The meta-analysis reported by Lai et al commonality of adverse effects in postanterior capsulotomies in the following order: headache (19.2\%), behavioral disorders (10.4\%), cognitive deficits (9.1\%), vestibular symptoms $(9.1 \%)$, mood changes $(6.5 \%)$, urinary incontinence (6.2\%), and others. Risk of severe permanent effects like brain cysts or brain edema (1.6\%) was small. ${ }^{7}$ Radiation doses of 120 to $160 \mathrm{~Gy}$ and precise shot/isocenter placement (like GVC) has brought down the risk of adverse radiation effects significantly.

\section{Limitations}

Our study has various limitations. Being a retrospective study, the accuracy of past clinical data obtained through medical records is nonuniform and lacking. Though admin- istered by experienced in-hospital clinical psychologists, YBOCS scores could not be standardized using interrater reliability. Follow-up data (frequency and intervals) were not consistent enough to comment on the changes in Y-BOCS scores at different time periods from the GKRS procedure. Additionally, objective measures of overall functioning like global assessment of functioning (GAF) scores, beck depression inventory (BDI), and state-trait anxiety inventory (STAI) - pre- and post-GKRS-may have been useful. Relatively rare disease entity, very small sample size, and wide range of variations in clinical and radiotherapeutic profiles in the study group undermine the significance of our results. No statistically significant results were obtained.

\section{Conclusion}

GKRS is a safe and effective noninvasive treatment modality for refractory OCD. Ventral anterior capsule is the preferred target site. Maximum radiation doses of 120 to $160 \mathrm{~Gy}$ are well tolerated. Extremely severe OCD cases fared poorer. Proper awareness about the availability and efficacy of GKRS in refractory OCD is required in India. 


\section{Conflict of Interest \\ None declared.}

\section{References}

1 American Psychiatric Association. Diagnostic and Statistical Manual of Mental Disorders. 5th ed. Philadelphia, PA: American Psychiatric Publishing; 2013

2 Ruscio AM, Stein DJ, Chiu WT, Kessler RC. The epidemiology of obsessive-compulsive disorder in the National Comorbidity Survey Replication. Mol Psychiatry 2010;15(01):53-63

3 Reddy YCJ, Arumugham SS. Are current pharmacotherapeutic strategies effective in treating OCD? Expert Opin Pharmacother 2020;21(08):853-856

4 Rathore PJ. Cognitive behavioral therapy and functional impairment in obsessive-compulsive disorder. Indian J Soc Psychiatry 2019;35:80

5 Balachander S, Arumugham SS, Srinivas D. Ablative neurosurgery and deep brain stimulation for obsessive-compulsive disorder. Indian J Psychiatry 2019;61(Suppl 1):S77-S84

6 Pauls DL, Abramovitch A, Rauch SL, Geller DA. Obsessive-compulsive disorder: an integrative genetic and neurobiological perspective. Nat Rev Neurosci 2014;15(06):410-424

7 Lai Y, Wang T, Zhang C, et al. Effectiveness and safety of neuroablation for severe and treatment-resistant obsessive-compulsive disorder: a systematic review and meta-analysis. J Psychiatry Neurosci 2020;45(05):356-369

8 Miguel EC, Lopes AC, McLaughlin NCR, et al. Evolution of gamma knife capsulotomy for intractable obsessive-compulsive disorder. Mol Psychiatry 2019;24(02):218-240

9 Chang KW, Jung HH, Chang JW. Magnetic resonance-guided focused ultrasound surgery for obsessive-compulsive disorders: potential for use as a novel ablative surgical technique. Front Psychiatry 2021;12:640832

10 Kumar KK, Appelboom G, Lamsam L, et al. Comparative effectiveness of neuroablation and deep brain stimulation for treatmentresistant obsessive-compulsive disorder: a meta-analytic study. J Neurol Neurosurg Psychiatry 2019;90(04):469-473

11 Tripathi M, Mukherjee K, Chhabra R, Radotra I, Singh AP, Radotra B. Gamma knife for obsessive compulsive disorder: can it be detrimental? Turk Neurosurg 2014;24(04):583-586

12 Goodman WK, Price LH. Assessment of severity and change in obsessive compulsive disorder. Psychiatr Clin North Am 1992;15 (04):861-869
13 D'Astous M, Cottin S, Roy M, Picard C, Cantin L. Bilateral stereotactic anterior capsulotomy for obsessive-compulsive disorder: long-term follow-up. J Neurol Neurosurg Psychiatry 2013;84(11): 1208-1213

14 Peker S, Samanci MY, Yilmaz M, Sengoz M, Ulku N, Ogel K. Efficacy and safety of gamma ventral capsulotomy for treatment-resistant obsessive-compulsive disorder: a single-center experience. World Neurosurg 2020;141:e941-e952

15 Rasmussen SA, Noren G, Greenberg BD, et al. Gamma ventral capsulotomy in intractable obsessive-compulsive disorder. Biol Psychiatry 2018;84(05):355-364

16 Lopes AC, Greenberg BD, Canteras MM, et al. Gamma ventral capsulotomy for obsessive-compulsive disorder: a randomized clinical trial. JAMA Psychiatry 2014;71(09):1066-1076

17 Gupta A, Shepard MJ, Xu Z, et al. An international radiosurgery research foundation multicenter retrospective study of gamma ventral capsulotomy for obsessive compulsive disorder. Neurosurgery 2019;85(06):808-816

18 Santos BFO, Gorgulho A, Saraiva CWC, et al. Understanding gamma ventral capsulotomy: Potential implications of diffusion tensor image tractography on target selectivity. Surg Neurol Int 2019;10:136

19 Rück C, Karlsson A, Steele JD, et al. Capsulotomy for obsessivecompulsive disorder: long-term follow-up of 25 patients. Arch Gen Psychiatry 2008;65(08):914-921

20 Lopes AC, Greenberg BD, Norén G, et al. Treatment of resistant obsessive-compulsive disorder with ventral capsular/ventral striatal gamma capsulotomy: a pilot prospective study. J Neuropsychiatry Clin Neurosci 2009;21(04):381-392

21 Gouvea F, Lopes A, Greenberg B, et al. Response to sham and active gamma ventral capsulotomy in otherwise intractable obsessivecompulsive disorder. Stereotact Funct Neurosurg 2010;88(03): 177-182

22 Kondziolka D, Flickinger JC, Hudak R. Results following gamma knife radiosurgical anterior capsulotomies for obsessive compulsive disorder. Neurosurgery 2011;68(01):28-32, discussion 23-3

23 Sheehan JP, Patterson G, Schlesinger D, Xu Z. Y knife surgery anterior capsulotomy for severe and refractory obsessive-compulsive disorder. J Neurosurg 2013;119(05):1112-1118

24 Spatola G, Martinez-Alvarez R, Martínez-Moreno N, et al. Results of Gamma Knife anterior capsulotomy for refractory obsessivecompulsive disorder: results in a series of 10 consecutive patients. J Neurosurg 2018;131(02):376-383 